\title{
Direct drainage of the basal vein of Rosenthal into the superior petrosal sinus: a literature review
}

\author{
Santiago Gutierrez ${ }^{1}$ Joe Iwanaga ${ }^{2,3}$, Aaron S. Dumont ${ }^{2}$, R. Shane Tubbs D $^{2,3,4,5,6}$ \\ ${ }^{1}$ Pontificia Universidad Javeriana, Bogotá, Colombia, ${ }^{2}$ Department of Neurosurgery, Tulane University School of Medicine, New Orleans, LA, \\ ${ }^{3}$ Department of Neurology, Tulane University School of Medicine, New Orleans, LA, USA, ${ }^{4}$ Department of Anatomical Sciences, St. George's \\ University, St. George's, Grenada, West Indies, ${ }^{5}$ Department of Structural \& Cellular Biology, Tulane University School of Medicine, New Orleans, LA, \\ ${ }^{6}$ Department of Neurosurgery and Ochsner Neuroscience Institute, Ochsner Health System, New Orleans, LA, USA
}

\begin{abstract}
An adult male was found to have a variation of the left basal vein of Rosenthal after presenting with complaints of headache and balance issues. In this case, the vein drained directly into the left superior petrosal sinus (SPS) instead of the great vein of Galen. Anatomical variation of the basal vein is likely due to embryonic development of the deep cerebral venous system as primitive structures either differentiate regress or further with age. These changes may result in the uncommon presentation seen in this case. To our knowledge, this is the first case that shows the basal vein drains into the SPS. The normal and variant anatomy of this vessel are discussed.
\end{abstract}

Key words: Basal vein of Rosenthal, Basal vein, Superior petrosal sinus, Tentorial sinus

Received July 25, 2020; Revised September 21, 2020; Accepted October 5, 2020

\section{Introduction}

The basal vein was first described in 1824 by FriederichChristian Rosenthal, a German surgeon and anatomist. The basal vein of Rosenthal (BVR) is a constant and large vein that collects blood from the basal part of the brain and structures of the cerebral hemispheres, diencephalon, and midbrain (Fig. 1) [1]. It arises from the junction of the anterior cerebral (ACV), deep middle cerebral (DMCV), and inferior striate veins (ISVs) among others and usually drains into the great vein of Galen (GVG). The basal vein's drainage and tributary branches are related to developmental events and embryology. Therefore, the physiological variations of the

\footnotetext{
Corresponding author:

Joe Iwanaga (i)

Department of Neurosurgery, Tulane University School of Medicine, New Orleans, LA 70112, USA

E-mail: iwanagajoeca@gmail.com
}

BVR and some pathological states such as aneurysms and vessel malformations are closely related [2]. The literature review will focus on the structural description of the variation and its relationship to embryology. We also present a case of a patient with a rare variation of the BVR and its direct terminal drainage into the superior petrosal sinus (SPS).

\section{Venous Drainage of the Brain}

The vast venous drainage of the brain can be grossly divided into two main systems: the superficial system comprising the superior cerebral veins, superior sagittal sinus, superficial middle cerebral vein, superior anastomotic vein (vein of Trolard), and the inferior anastomotic vein (vein of Labbé) (Fig. 2); and the deep venous or galenic system (Fig. 3) that mainly comprises the GVG, the internal cerebral veins (ICVs), the BVR, and the inferior and superior sagittal sinuses with their tributaries [3]. All the aforementioned deep structures join into the GVG, which then joins the straight sinus to drain into the torcular Herophili; this finally unites 


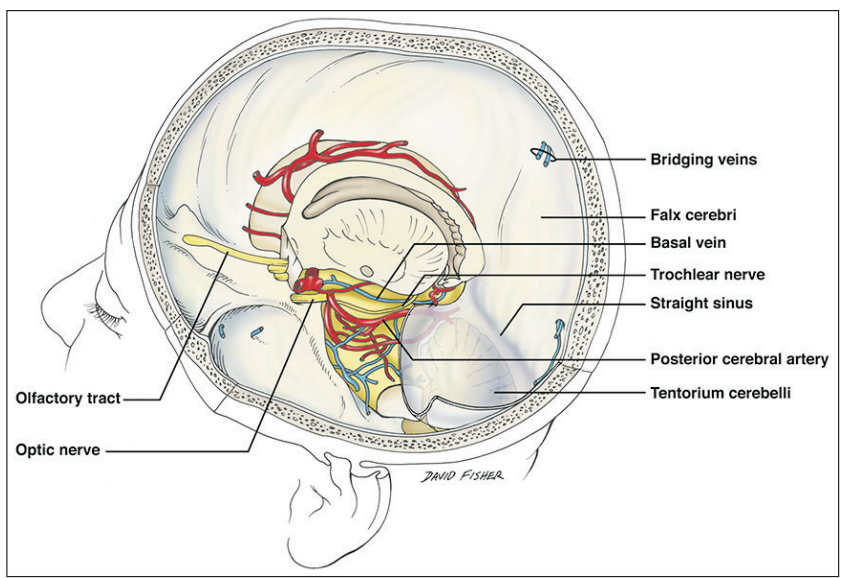

Fig. 1. Lateral view of the cranium noting the course of the basal vein in the ambient cistern.

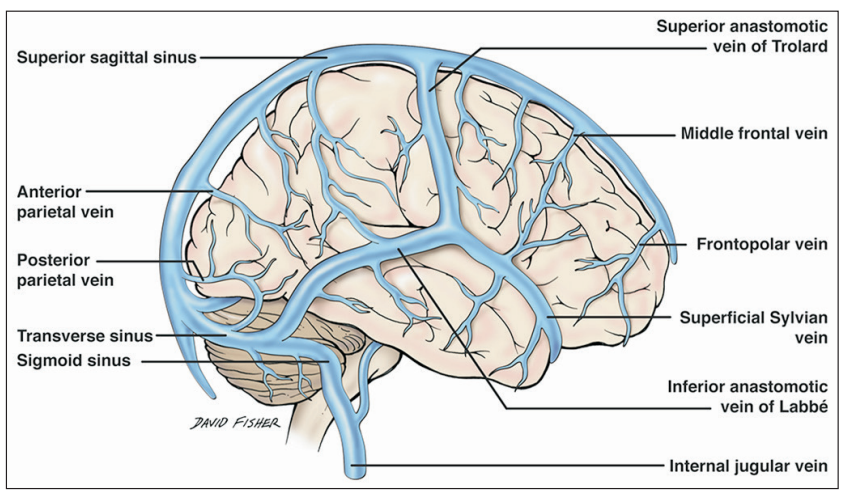

Fig. 2. Superficial venous system of the brain.

the superficial and deep systems into the final path of drainage via the transverse and sigmoid sinuses. Other dural venous sinuses such as the cavernous drain into the SPS, which in turn drains into the transverse sinus.

\section{Anatomical Description of the Basal Vein of Rosenthal}

The origin of the BVR is located in the anterior perforated substance and is formed by the junction of the ACV, DMCV, medial and inferior frontal, and ISVs (Fig. 3) $[1,4$, 5]. Several other tributaries have been mentioned such as the hippocampal, inferior choroidal, inferior ventricular, peduncular, posterior thalamic, lateral mesencephalic, pontine, precentral and quadrigeminal veins [5]. Therefore, the BVR is responsible for draining a large portion of all the basal brain structures such as the pons, and the mesencephalon, diencephalon and cortical regions. The largest tributary to

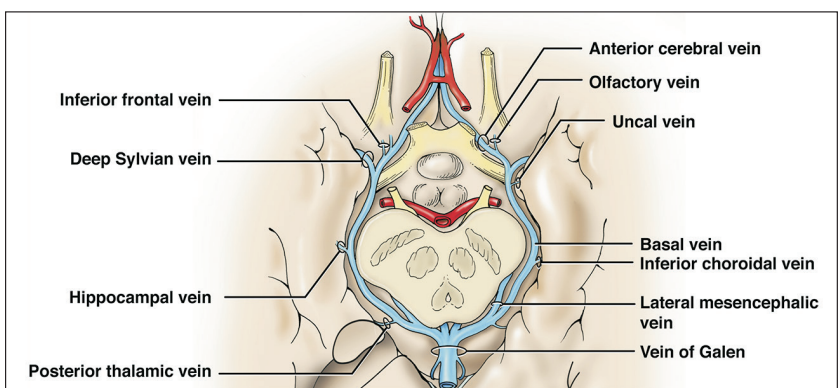

Fig. 3. Deep (galenic) venous system of the brain.

the BVR is the DMCV [1], though the inferior choroidal vein can also be substantial when connected.

The BVR follows an anteroposterior, inferosuperior and external sigmoid course. In almost $100 \%$ of cases, it traverses the crural and ambient cisterns along a course parallel to the posterior cerebral artery in a superomedial fashion [5]. Running between the cerebral peduncles and the temporal lobe, the BVR can be segmented into three portions: (1) The anterior or striate segment; (2) The middle or peduncular segment, which can in turn be divided into two subdivisions, anterior and posterior; and (3) The posterior or mesencephalic segment, which is also divided into two subdivisions, tegmental and terminal $[1,4]$.

The first, anterior or striate segment begins with the junction of the ACV, DMCV, and ISV. It follows an anterior to posterior, superior to inferior and lateral to medial course outlining the uncus, ending in the most medial point of the BVR where it is placed in the anterior surface of the cerebral peduncle. The first tributaries to this first segment include the DMCV and the ISV. The ACV then joins the vein, and so do the olfactory and posterior fronto-orbital veins and several twigs from the optic chiasm.

The second, middle or peduncular segment follows an arched silhouette with lateral convexity and an inferior to superior course. It can be divided into two portions by its relationship to the cerebral peduncle. The anterior portion of the peduncular segment is applied to the peduncular anterior surface but ends when the vein separates from it to become the posterior segment, laterally into the ambient cistern where relates more closely to the optic tract. This segment ends when the BVR traverses the lateral mesencephalic sulcus just before the lateral mesencephalic vein joins it. Tributaries described as joining the BV in this portion are the peduncular, inferior thalamic, and inferior ventricular veins and twigs from the optic tract, hypothalamus, and 


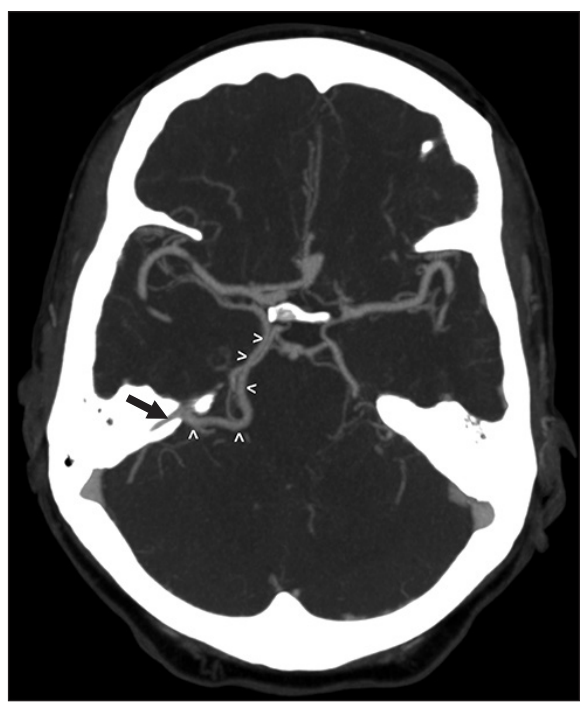

Fig. 4. Axial CTA windowed to illustrate the basal vein (white arrowheads) draining posteriorly into the SPS (black arrow) (Courtesy of Dr. Joel Curé). CTA, computed tomography angiography; SPS, superior petrosal sinus.

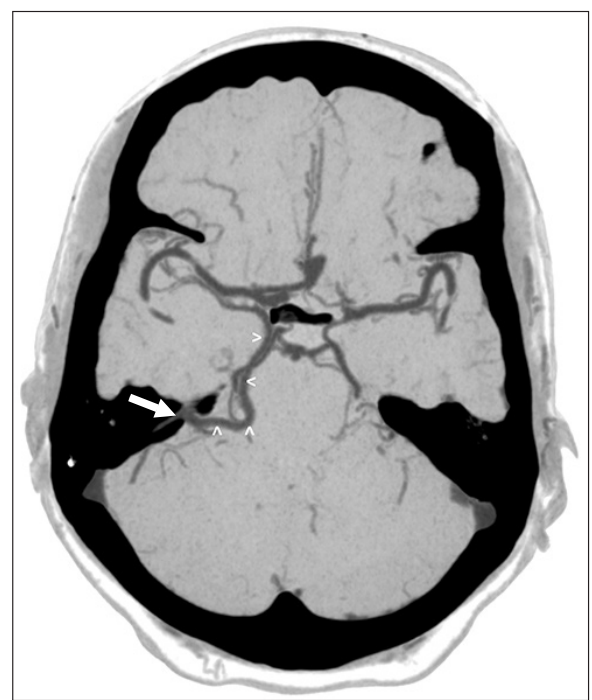

Fig. 5. Axial CTA windowed to illustrate the basal vein (white arrowheads) draining posteriorly into the superior petrosal sinus (SPS) (white arrow) (Courtesy of Dr. Joel Curé). CTA, computed tomography angiography; SPS, superior petrosal sinus.

temporal lobe.

The third portion, also called the posterior or mesencephalic portion, starts distally to the lateral mesencephalic sulcus and proximal to the joining of the lateral mesencephalic vein [1]. It is the highest portion and follows an ascendant, lateral-to-medial trajectory until it joins the ICVs into the GVG. As we will discuss below, several variants in the

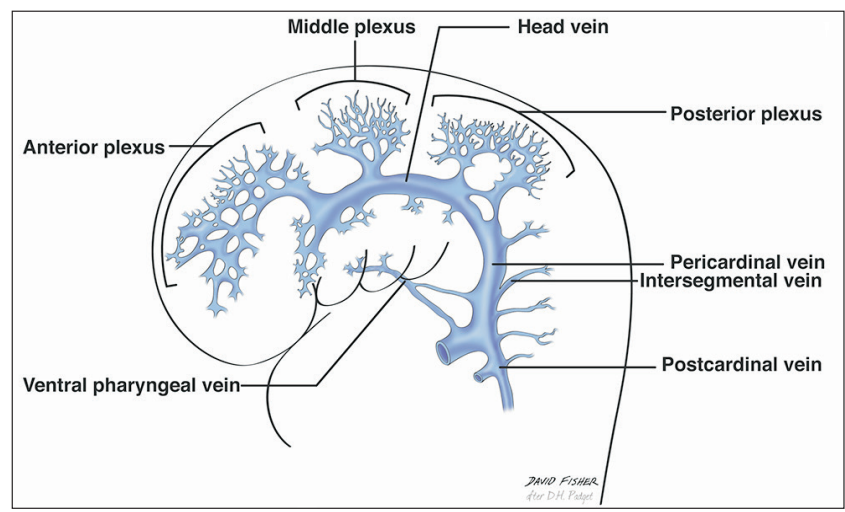

Fig. 6. Early embryological development of the veins of the brain noting the anterior, middle and posterior plexuses.

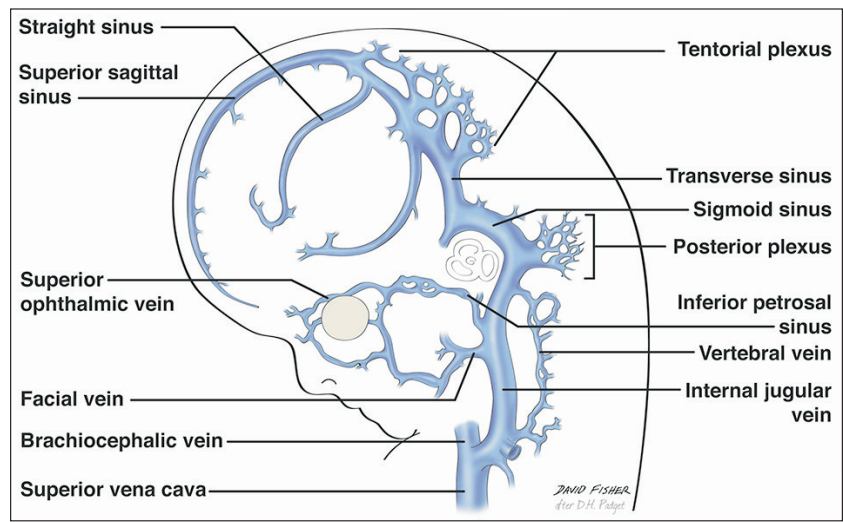

Fig. 7. Later embryological development of the veins of the brain noting the tentorial plexus.

termination of the vein have been described with patterns arising from early developmental stages. These termination patterns include the straight sinus, the torcular Herophili and the transverse sinus $[2,4,6]$. Tributaries related to this segment are the lateral mesencephalic [7], thalamogeniculate, and medial aspects of temporal veins.

\section{Case Presentation}

An elderly adult male presented with headaches and balance issues over one week and was evaluated for intracranial pathology. There was no recent history of disease or illness but a concern for intracranial vascular pathology, therefore, a computed tomography angiography (CTA) was obtained. This demonstrated a left basal vein that traveled posteriorly to empty into the left SPS (Figs. 4, 5). No aneurysms or other intracranial pathology such as arteriovenous malformation (AVM), dural arteriovenous fistula or masses were noted. 
No other anatomical variations of the intracranial anatomy were noted in this patient. The right-sided basal vein formed normally and drained into the vein of Galen, which then drained into the straight sinus. The anatomical variation identified was felt to be incidental and not related to the patient's presenting symptoms.

\section{Embryological Considerations}

Venous drainage of the skull and brain has an intricate embryological origin, as described by several authors such as Padgett [2] in 1956. She wrote a thorough description of the development of the brain venous system in eight stages during the first three months of intrauterine life. Both the superficial and deep systems start the first stages of development with three primary stems called the anterior, middle, and posterior plexuses (dural vessels) (Fig. 6). These stems drain into the so-called primary head sinus (stage two in Padget's description), which in turn joins the ventral pharyngeal vein to form the anterior cardinal vein, which later differentiates into the jugular vein. The three primary dural plexuses then give rise to the primary pial veins, listed as the telencephalic, diencephalic, mesencephalic, metencephalic, and myelencephalic veins $[2,4,5]$.

Suzuki et al. [6] stated that the formation of the BVR depends mainly on four components derived from the telencephalic and mesencephalic veins: The deep telencephalic, ventral diencephalic, and dorsal diencephalic veins join the metencephalic vein to form the BVR at the 60 to $80 \mathrm{~mm}$ stage. Each of these structures drains via one or more of five routes: the GVG, cavernous sinus, SPS via the peduncular vein or lateral mesencephalic vein, and the transverse or straight sinus via the tentorium. As a general rule, the dural veins and sinuses do not drain into the BVR; its tributaries tend to have predominantly pial origins $[1,2]$.

The longitudinal secondary anastomoses between the primitive pial plexuses that give rise to the BVR are the same as those forming the tentorial sinus at the $18 \mathrm{~mm}$ stage [1]. Therefore, the BVR has many possible variations. Variations among these longitudinal anastomoses can produce a fragmented BVR and also predispose to the survival of remnants of the primitive tentorial plexus that can in some cases result in clinical manifestations (see clinical considerations) [8].

Regarding the tentorial sinus, the anterior stem of the primary head sinus drains the venous territories of the telencephalic, diencephalic and mesencephalic veins (14 mm stage).
This anterior stem later joins the middle stem to become the primitive tentorial plexus (sinus) (Fig. 7) (18 mm stage), which in turn slowly differentiates to drain the supratentorial and infratentorial territories. Therefore, the tentorial plexus and future BVR can receive tributaries from both the supratentorial and infratentorial areas. Remnant tentorial sinuses or lacunae can be observed in $12.1 \%$ to $86 \%$ of the general population $[4,8]$. These remnant tentorial patterns should be studied in conjunction with variations of the BVR as they share aspects of presentation and invariably derive from the telencephalic and diencephalic veins $[4,9]$.

Suzuki et al. [6] presented a case series of 500 sides using three-dimensional (3D) computed tomography (CT) angiography imaging and found that the BVR had a connection to the GVG in $87 \%$ of cases. The configuration of the BVR that is usually described was found in only $55.4 \%$ of the sides studied. The authors described variations based on the anatomical BVR segments described by Huang et al. [1]. They reported the outflow of the fragmented or hypoplastic BVR into the cavernous sinus, lateral mesencephalic vein to the SPS, pedicular veins and either the lateral or medial tentorial sinus.

In 2005, Chung et al. [4] reviewed the variations and efferent pathways of the BVR in 82 hemispheres using a classification system based on their embryological origin, supporting Padget's hypothesis. They grouped them into four categories: Group A (43\% and most common) for the telencephalic tributaries including the uncal, inferior frontal, and inferior striatal veins; Group B (35\%) for the diencephalic tributaries including the inferior ventricular and peduncular veins; Group C (11\%) for the tegmental bridging group including the longitudinal lateromesencephalic anastomoses; and group D (5\% and least common) for the superior vermian and internal occipital veins. The remaining $6 \%$ were unclassified. The same authors found that up to $10 \%$ of the cases had no galenic connection and there was an embryonic tentorial sinus in up to $12 \%$. Watanabe et al. [10] also presented a classification of BVR variations depending on the mainstream drainage but not considering the embryological origins. They established a clinical correlation of variants of the BVR with nonaneurysmal subarachnoid hemorrhage [10].

Connections between the BVR and the SPS are usually established via the peduncular and lateral mesencephalic veins in the adult configuration. Interestingly, our presented case exhibited a direct terminal connection between the BVR and SPS with no remnant tentorial sinus. This kind of direct con- 
nection has not been reported in the literature to our knowledge. However, it can be explained by Padgett's and Chung's accounts.

\section{Radiological Assessment of BVR Variations}

Assessing the venous drainage is crucial for preoperative skull base surgery planning $[6,11]$, especially information regarding the drainage pathway of the BVR. Because there are so many variations, radiological assessment constitutes a clinical challenge for surgeons. The available techniques include digital subtraction angiography (DSA), 3D CT angiography, CT venography, magnetic resonance (MR) venography, maximum intensity projection (MIP), and multiplanar reconstruction (MPR). Each of these has advantages and disadvantages for assessing the venous vasculature. For instance, angiography provides limited delineation of the venous phases and cannot provide structural information in 3D. A 3D CT angiography provides limited detection of vessels less than $1 \mathrm{~mm}$ in diameter but gives a detailed assessment of the anatomical relationships regarding the arterial and bony structures.

For venography, the CT protocol provides a better delineation of venous structures than any form of MRI. MIP and MPR delineate small structures with better definition than the aforementioned techniques. Thus, volume information is more precise than with 3D CT angiography [12]. All individual radiological techniques are limited in the assessments of BVR morphology they provide, but this can be mitigated when the techniques are combined. Suzuki et al. [6] stated that the discrimination of the BVR by MPR and MIP ranged between $66 \%$ and $96 \%$, while 3D CT angiography with MIP identified the vein in all the sides studied but failed to identify some of the narrow and hypoplastic anastomoses and tributaries of the BVR [13].

\section{Clinical and Surgical Considerations}

Several clinical indications have been linked to variations of the BVR. For instance, remote cerebellar hemorrhages have a well-described relationship to BVR variations [14]. Pediatric sinovenous thrombosis is a rare and severe disorder that has also been linked to venous variations including those of the deep venous system [14]. Ract et al. [15] published a case report of a patient presenting with extensive basal ganglia edema caused by a carotid-cavernous fistula in the presence of a hypoplastic second segment of the BVR. Cases of AVMs with drainage to the BVR were reported in 2016 by Nishino et al. [16]. Also in 2016, Cannizzaro et al. [17] found that patients with dural arteriovenous fistulae involving the lateral mesencephalic vein can present retrograde flow into the BVR $[18,19]$.

Several surgical skull base approaches such as the occipital transtentorial (Dandy's) and infratentorial supracerebellar (Stein's) approaches provide a surgical corridor to the pineal region. The occipital transtentorial has several advantages over Stein's approach because it provides superior visibility over the tentorial notch, which facilitates the resection of large tumors [20]. However, both approaches require the retraction and manipulation of deep venous structures such as the GVG, the ICV and the BVR, and the retraction of the GVG and its tributaries can lead to hemorrhagic infarction. Furthermore, dural incisions during the occipital transtentorial approaches can be problematic if there is a remnant of the lateral or medial tentorial sinuses. Iatrogenic lesions in the deep venous system can cause severe sequelae such as consciousness disturbances, status epilepticus, visual impairment due to occipital lobe injury, hemiplegia and death. This is why careful assessment of the vascular structures of the skull base is imperative [13]. For pterional approaches to the skull base, assessment of the anterior segment of BVR is needed to preclude injury. For example, if the first segment of the BVR drains into the lateral mesencephalic or peduncular vein (both related to SPS), venous connections between the middle and posterior fossae should be suspected when subtemporal approaches are performed [6]. For interventional neuroradiology approaches, understanding of the variations of the basal venous drainage of the skull base is imperative [4].

\section{Conclusion}

Anatomical variations related to BVR are numerous and can present on its tributaries and its drainage pathway. These variations, determined by embryological development, are derived explicitly from the telencephalic, diencephalic and mesencephalic primitive pial veins and their secondary longitudinal anastomoses. They should be classified by considering the developmental aspects and not just structurally, and must be assessed properly when a preoperative skull base procedure is planned. The presented case report of a direct connection between the BVR and the SPS in the absence of tentorial sinuses is, to our knowledge, the first of its kind to 
be reported in the literature.

\section{ORCID}

\author{
Santiago Gutierrez: \\ https://orcid.org/0000-0003-1326-8308 \\ Joe Iwanaga: https://orcid.org/0000-0002-8502-7952 \\ Aaron S. Dumont: https://orcid.org/0000-0002-8077-8992 \\ R. Shane Tubbs: https://orcid.org/0000-0003-1317-1047
}

\section{Author Contributions}

Conceptualization: JI, RST. Data acquisition: SG, JI, RST. Data analysis or interpretation: SG, ASD. Drafting of the manuscript: SG, JI. Critical revision of the manuscript: ASD, RST. Approval of the final version of the manuscript: all authors.

\section{Conflicts of Interest}

No potential conflict of interest relevant to this article was reported.

\section{References}

1. Huang YP, Wolf BS. The basal cerebral vein and its tributaries. In: Newton TH, Potts DG, editors. Radiology of the Skull and Brain, Vol. 2: Angiography. Saint Louis: Mosby; 1974. p.2111-54.

2. Padget $\mathrm{DH}$. The cranial venous system in man in reference to development, adult configuration, and relation to the arteries. Am J Anat 1956;98:307-55.

3. Uddin MA, Haq TU, Rafique MZ. Cerebral venous system anatomy. J Pak Med Assoc 2006;56:516-9.

4. Chung JI, Weon YC. Anatomic variations of the deep cerebral veins, tributaries of basal vein of rosenthal: embryologic aspects of the regressed embryonic tentorial sinus. Interv Neuroradiol 2005;11:123-30.

5. Tubbs RS, Loukas M, Louis RG Jr, Shoja MM, Askew CS, Phantana-Angkool A, Salter EG, Oakes WJ. Surgical anatomy and landmarks for the basal vein of rosenthal. J Neurosurg 2007;106:900-2.

6. Suzuki Y, Ikeda H, Shimadu M, Ikeda Y, Matsumoto K. Variations of the basal vein: identification using three-dimensional CT angiography. AJNR Am J Neuroradiol 2001;22:670-6.

7. Ardeshiri A, Ardeshiri A, Tonn JC, Winkler PA. Microsurgical anatomy of the lateral mesencephalic vein and its mean- ing for the deep venous outflow of the brain. Neurosurg Rev 2006;29:154-8; discussion 158.

8. Muthukumar N, Palaniappan P. Tentorial venous sinuses: an anatomic study. Neurosurgery 1998;42:363-71.

9. San Millán Ruíz D, Fasel JH, Reverdin A, Gailloud P. Bilateral tentorial sinus drainage of the basal vein (of Rosenthal). Clin Anat 2003;16:264-8.

10. Watanabe A, Hirano K, Kamada M, Imamura K, Ishii N, Sekihara Y, Suzuki Y, Ishii R. Perimesencephalic nonaneurysmal subarachnoid haemorrhage and variations in the veins. Neuroradiology 2002;44:319-25.

11. Linn J, Strueder K, Winkler PA, Brückmann H. The value of contrast-enhanced MR angiography in visualization of the basal vein of rosenthal and the lateral mesencephalic vein and their intra- and interindividual variations. Skull Base 2009;19:A293.

12. Karakas AB, Govsa F, Ozer MA, Eraslan C. 3D brain imaging in vascular segmentation of cerebral venous sinuses. J Digit Imaging 2019;32:314-21.

13. Suzuki Y, Nakajima M, Ikeda H, Abe T. Three-dimensional computed tomography angiography of the galenic system for the occipital transtentorial approach. Neurol Med Chir (Tokyo) 2005;45:387-93; discussion 393-4.

14. Kouzmitcheva E, Andrade A, Muthusami P, Shroff M, MacGregor DL, deVeber G, Dlamini N, Moharir M. Anatomical venous variants in children with cerebral sinovenous thrombosis. Stroke 2019;50:178-80.

15. Ract I, Drier A, Leclercq D, Sourour N, Gabrieli J, Yger M, Nouet A, Dormont D, Chiras J, Clarençon F. Extensive basal ganglia edema caused by a traumatic carotid-cavernous fistula: a rare presentation related to a basal vein of Rosenthal anatomical variation. J Neurosurg 2014;121:63-6.

16. Nishino K, Hasegawa H, Morita K, Fukuda M, Ito Y, Fujii Y, Sato M. Clinical characteristics of arteriovenous malformations in the cerebellopontine angle cistern. J Neurosurg 2017;126:608.

17. Cannizzaro D, Rammos SK, Peschillo S, El-Nashar AM, Grande AW, Lanzino G. The lateral mesencephalic vein: surgical anatomy and its role in the drainage of tentorial dural arteriovenous fistulae. World Neurosurg 2016;85:163-8.

18. Kiyosue H, Mori H, Sagara Y, Hori Y, Okahara M, Nagatomi $\mathrm{H}$, Abe T. Basal cerebral venous drainage from cavernous sinus dural arteriovenous fistulas. Neuroradiology 2009;51:175-81.

19. Shimada R, Kiyosue H, Tanoue S, Mori H, Abe T. Superior petrosal sinus: hemodynamic features in normal and cavernous sinus dural arteriovenous fistulas. AJNR Am J Neuroradiol 2013;34:609-15.

20. Porter RW, Detwiler PW, Spetzler RF. Surgical approaches to the brain stem. Oper Tech Neurosurg 2000;3:114-23. 